\title{
UNIQUENESS OF SOLUTION AND FULLY DISCRETE SCHEME TO NONLINEAR INTEGRO-DIFFERENTIAL AVERAGED MODEL WITH SOURCE TERMS
}

\author{
T. JANGVELADZE, Z. KIGURADZE, AND M. KRATSASHVILI
}

Received 14 March, 2018

\begin{abstract}
Uniqueness of solution and finite difference scheme of corresponding initial-boundary value problem for one nonlinear partial integro-differential averaged model with source terms are studied. Mentioned model is based on Maxwell system which describes electromagnetic field penetration into a substance. Mixed boundary condition is considered. Large time behavior of solution is fixed too. Convergence of the fully discrete scheme is proved. Wider class of nonlinearity is studied than one has been investigated before.
\end{abstract}

2010 Mathematics Subject Classification: 65N06; 45K05; 35K55

Keywords: Maxwell equations, system of nonlinear integro-differential equations, uniqueness, asymptotic behavior, finite difference scheme

\section{INTRODUCTION}

Investigating many applied problems in nature we are facing to nonlinear integrodifferential models which contain derivatives of several variables. Numerous publications deal with the study of integro-differential equations of various kinds (see, for example, the bibliography in [6], [8]). Integro-differential models arise for example at the mathematical simulation of process of a magnetic field penetration into a medium whose electro-conductivity depends on temperature. Numerous works are dedicated to the investigation of Maxwell equations describing above-mentioned process (see, for example, [12], [19], [20] and references therein). In a quasi-stationary case the corresponding system of Maxwell equations [12] can be rewritten in the following form [5]:

$$
\frac{\partial H}{\partial t}=-\operatorname{rot}\left[a\left(\int_{0}^{t}|\operatorname{rot} H|^{2} d \tau\right) \operatorname{rot} H\right]
$$

The third author thanks Shota Rustaveli National Science Foundation (project PhDF2016_19) for the financial support. 
where $H=\left(H_{1}, H_{2}, H_{3}\right)$ is a vector of the magnetic field and function $a=a(S)$ is defined for $S \in[0, \infty)$.

If vector of magnetic field has the form $H=(0, U, V)$, where $U=U(x, t), V=$ $V(x, t)$, then from (1.1) we get the following system of nonlinear parabolic integrodifferential equations:

$$
\frac{\partial U}{\partial t}=\frac{\partial}{\partial x}\left[a(S) \frac{\partial U}{\partial x}\right], \quad \frac{\partial V}{\partial t}=\frac{\partial}{\partial x}\left[a(S) \frac{\partial V}{\partial x}\right],
$$

where

$$
S(x, t)=\int_{0}^{t}\left[\left(\frac{\partial U}{\partial x}\right)^{2}+\left(\frac{\partial V}{\partial x}\right)^{2}\right] d \tau .
$$

Study of the models of type (1.1) have begun in [5]. In that work, in particular, based on Galerkin modified method and compactness arguments as in [17] for nonlinear parabolic equations the theorems of existence of solution of the initial-boundary value problem with first kind boundary conditions for scalar and one-dimensional space case when $a(S)=1+S$ and uniqueness for more general cases are proven. One-dimensional scalar variant for the case $a(S)=(1+S)^{p}, 0<p \leq 1$ is studied in [3]. Investigations for multi-dimensional space cases at first was carried out in [4]. Multidimensional space cases are also discussed in [1], [13]. Asymptotic behavior as $t \rightarrow \infty$ of solutions of initial-boundary value problems for (1.1) type models are studied in [8], [9], [10], [15], [23] and in a number of other works as well. In those works main attention is paid to one-dimensional cases. Finite element analogues and Galerkin method algorithm as well as settling of semi-discrete and finite difference schemes for (1.1) type one-dimensional integro-differential models are studied in [2], [7], [8], [9], [15], [18], [22], [23], [24] and in the other works as well for the linear case of diffusion coefficient, i.e. $a(S)=1+S$.

Some generalization of the system of type (1.1) is proposed by Prof. G. I. Laptev [14]. In particular, in some physical assumptions, the process of penetration of the magnetic field into a material is modeled by so-called averaged integro-differential model, the (1.2), (1.3) type analog of which have the following form:

$$
\frac{\partial U}{\partial t}=a(S) \frac{\partial^{2} U}{\partial x^{2}}, \quad \frac{\partial V}{\partial t}=a(S) \frac{\partial^{2} V}{\partial x^{2}},
$$

where

$$
S(t)=\int_{0}^{t} \int_{0}^{1}\left[\left(\frac{\partial U}{\partial x}\right)^{2}+\left(\frac{\partial V}{\partial x}\right)^{2}\right] d x d \tau .
$$

The literature on the questions of existence, uniqueness, regularity, asymptotic behavior of the solutions and numerical resolution of the initial-boundary value problems to the (1.2), (1.3) and (1.4), (1.5) type models and models like them is very rich (see, for example, [5], [6], [7], [8], [14], [15], [16], [18] and references therein). The 
asymptotic behavior and numerical solution of two-dimensional case for the (1.4), (1.5) type averaged integro-differential system is considered for example in [11], [24].

Our aim is to investigate system of nonlinear integro-differential equations (1.4), (1.5) with source terms. Uniqueness and large time behavior of solution of initialboundary value problem with mixed boundary condition as well as convergence of corresponding fully discrete scheme is studied. In the present note we consider new class of nonlinearity considering more general cases of the diffusion coefficient $a=$ $a(S)$.

\section{UNIQUENESS AND ASYMPTOTIC BEHAVIOR OF SOLUTION AS $t \rightarrow \infty$}

In the cylinder $[0,1] \times[0, \infty)$ let us consider the following initial-boundary value problem:

$$
\begin{gathered}
\frac{\partial U}{\partial t}-a\left(\int_{0}^{t} \int_{0}^{1}\left[\left(\frac{\partial U}{\partial x}\right)^{2}+\left(\frac{\partial V}{\partial x}\right)^{2}\right] d x d \tau\right) \frac{\partial^{2} U}{\partial x^{2}}+g(U)=f_{1}(x, t), \\
\frac{\partial V}{\partial t}-a\left(\int_{0}^{t} \int_{0}^{1}\left[\left(\frac{\partial U}{\partial x}\right)^{2}+\left(\frac{\partial V}{\partial x}\right)^{2}\right] d x d \tau\right) \frac{\partial^{2} V}{\partial x^{2}}+g(V)=f_{2}(x, t), \\
U(0, t)=V(0, t)=\left.\frac{\partial U(x, t)}{\partial x}\right|_{x=1}=\left.\frac{\partial V(x, t)}{\partial x}\right|_{x=1}=0, \\
U(x, 0)=U_{0}(x), \quad V(x, 0)=V_{0}(x),
\end{gathered}
$$

where $a=a(S), g, f_{1}, f_{2}, U_{0}$ and $V_{0}$ are given functions of their arguments.

The following statement takes place.

Theorem 1. If $a=a(S) \geq a_{0}=$ Const $>0, a^{\prime}(S) \geq 0, a^{\prime \prime}(S) \leq 0, g$ is monotonically increased function, $U_{0}, V_{0} \in H^{1}(0,1), U_{0}(0)=V_{0}(0)=\left.\frac{d U_{0}(x)}{d x}\right|_{x=1}=$ $\left.\frac{d V_{0}(x)}{d x}\right|_{x=1}=0, f_{1}, f_{2}, \frac{\partial f_{1}}{\partial x}, \frac{\partial f_{2}}{\partial x} \in L_{2}(Q)$ and problem (2.1) - (2.3) has a solution then it is unique and exponential stabilization of solution as $t \rightarrow \infty$ takes place.

Here we use usual $L_{2}$ and Sobolev $H^{1}$ spaces.

To prove the uniqueness of solution we assume that there exist two different $(\bar{U}, \bar{V})$ and $\overline{\bar{U}}, \overline{\bar{V}})$ solutions of problem (2.1) - (2.3) and introduce the differences $Z=$ $\overline{\bar{U}}-\bar{U}$ and $W=\overline{\bar{V}}-\bar{V}$. To show that $Z=W \equiv 0$ the methodology of proving the convergence theorem, which is given in the next section, monotone growth feature of function $g$ and the following identity is mainly used:

$$
\left\{a\left(\int_{0}^{t} \int_{0}^{1}\left[\left(\frac{\partial \overline{\bar{U}}}{\partial x}\right)^{2}+\left(\frac{\partial \overline{\bar{V}}}{\partial x}\right)^{2}\right] d x d \tau\right) \frac{\partial \overline{\bar{U}}}{\partial x}\right.
$$




$$
\begin{gathered}
\left.-a\left(\int_{0}^{t} \int_{0}^{1}\left[\left(\frac{\partial \bar{U}}{\partial x}\right)^{2}+\left(\frac{\partial \bar{V}}{\partial x}\right)^{2}\right] d x d \tau\right) \frac{\partial \bar{U}}{\partial x}\right\}\left(\frac{\partial \overline{\bar{U}}}{\partial x}-\frac{\partial \bar{U}}{\partial x}\right) \\
+\left\{a\left(\int_{0}^{t} \int_{0}^{1}\left[\left(\frac{\partial \overline{\bar{U}}}{\partial x}\right)^{2}+\left(\frac{\partial \overline{\bar{V}}}{\partial x}\right)^{2}\right] d x d \tau\right) \frac{\partial \overline{\bar{V}}}{\partial x}\right. \\
\left.-a\left(\int_{0}^{t} \int_{0}^{1}\left[\left(\frac{\partial \bar{U}}{\partial x}\right)^{2}+\left(\frac{\partial \bar{V}}{\partial x}\right)^{2}\right] d x d \tau\right) \frac{\partial \bar{V}}{\partial x}\right\}\left(\frac{\partial \overline{\bar{V}}}{\partial x}-\frac{\partial \bar{V}}{\partial x}\right) \\
=\int_{0}^{1} \frac{d}{d \mu} a\left(\int_{0}^{t} \int_{0}^{1}\left\{\left[\frac{\partial \bar{U}}{\partial x}+\mu\left(\frac{\partial \overline{\bar{U}}}{\partial x}-\frac{\partial \bar{U}}{\partial x}\right)\right]^{2}+\left[\frac{\partial \bar{V}}{\partial x}+\mu\left(\frac{\partial \overline{\bar{V}}}{\partial x}-\frac{\partial \bar{U}}{\partial x}\right)\right]^{2}\right\} d x d \tau\right) \\
\times\left[\frac{\partial \bar{U}}{\partial x}+\mu\left(\frac{\partial \overline{\bar{U}}}{\partial x}-\frac{\partial \bar{U}}{\partial x}\right)\right] d \mu\left(\frac{\partial \overline{\bar{U}}}{\partial x}-\frac{\partial \bar{U}}{\partial x}\right) \\
+\int_{0}^{1} \frac{d}{d \mu} a\left(\int_{0}^{t} \int_{0}^{1}\left\{\left[\frac{\partial \bar{U}}{\partial x}+\mu\left(\frac{\partial \overline{\bar{U}}}{\partial x}-\frac{\partial \bar{U}}{\partial x}\right)\right]^{2}+\left[\frac{\partial \bar{V}}{\partial x}+\mu\left(\frac{\partial \overline{\bar{V}}}{\partial x}-\frac{\partial \bar{U}}{\partial x}\right)\right]^{2}\right\} d x d \tau\right) \\
\times\left[\frac{\partial \bar{V}}{\partial x}+\mu\left(\frac{\partial \overline{\bar{V}}}{\partial x}-\frac{\partial \bar{V}}{\partial x}\right)\right] d \mu\left(\frac{\partial \overline{\bar{V}}}{\partial x}-\frac{\partial \bar{V}}{\partial x}\right) .
\end{gathered}
$$

For obtaining stabilization of solution stated in the Theorem 1 the method of apriori estimates based on analogical methodology given in [7] is used and large time behavior of solution is obtained.

\section{CONVERGENCE OF THE FULLY DisCRETE SCHEME}

In the rectangle $Q_{T}=[0,1] \times[0, T]$, where $T$ is a positive constant, let us consider again problem (2.1) - (2.3). On $Q_{T}$ let us introduce a net with mesh points denoted by $\left(x_{i}, t_{j}\right)=(i h, j \tau)$, where $i=0,1, \ldots, M ; j=0,1, \ldots, N$ with $h=1 / M, \tau=T / N$. The initial line is denoted by $j=0$. The discrete approximation at $\left(x_{i}, t_{j}\right)$ is designed by $\left(u_{i}^{j}, v_{i}^{j}\right)$ and the exact solution to the problem (2.1) - (2.3) by $\left(U_{i}^{j}, V_{i}^{j}\right)$. We will use the following known notations [21] of forward and backward derivatives:

$$
r_{x, i}^{j}=\frac{r_{i+1}^{j}-r_{i}^{j}}{h}, \quad r_{\bar{x}, i}^{j}=\frac{r_{i}^{j}-r_{i-1}^{j}}{h}, \quad r_{t, i}^{j}=\frac{r_{i}^{j+1}-r_{i}^{j}}{\tau}
$$

and inner products and norms:

$$
\begin{gathered}
\left(r^{j}, y^{j}\right)=h \sum_{i=1}^{M-1} r_{i}^{j} y_{i}^{j}, \quad\left(r^{j}, y^{j}\right]=h \sum_{i=1}^{M} r_{i}^{j} y_{i}^{j}, \\
\left.\left\|r^{j}\right\|=\left(r^{j}, r^{j}\right)^{1 / 2}, \quad \| r^{j}\right] \mid=\left(r^{j}, r^{j}\right]^{1 / 2} .
\end{gathered}
$$


For problem (2.1) - (2.3) let us consider the following finite difference scheme:

$$
\begin{gathered}
\frac{u_{i}^{j+1}-u_{i}^{j}}{\tau}-a\left(\tau h \sum_{k=1}^{j+1} \sum_{\ell=1}^{M}\left[\left(u_{\bar{x}, \ell}^{k}\right)^{2}+\left(v_{\bar{x}, \ell}^{k}\right)^{2}\right]\right) u_{\bar{x} x, i}^{j+1}+g\left(u_{i}^{j+1}\right)=f_{1, i}^{j}, \\
\frac{v_{i}^{j+1}-v_{i}^{j}}{\tau}-a\left(\tau h \sum_{k=1}^{j+1} \sum_{\ell=1}^{M}\left[\left(u_{\bar{x}, \ell}^{k}\right)^{2}+\left(v_{\bar{x}, \ell}^{k}\right)^{2}\right]\right) v_{\bar{x} x, i}^{j+1}+g\left(v_{i}^{j+1}\right)=f_{2, i}^{j}, \\
i=1,2, \ldots, M-1 ; j=0,1, \ldots, N-1, \\
u_{0}^{j}=v_{0}^{j}=u_{\bar{x}, M}^{j}=v_{\bar{x}, M}^{j}=0, \quad j=0,1, \ldots, N, \\
u_{i}^{0}=U_{0, i}, v_{i}^{0}=V_{0, i}, i=0,1, \ldots, M .
\end{gathered}
$$

Multiplying equations in (3.1) scalarly by $u_{i}^{j+1}$ and $v_{i}^{j+1}$ respectively, it is not difficult to get the inequalities:

$$
\left.\left.\left\|u^{n}\right\|^{2}+\sum_{j=1}^{n} \| u_{\bar{x}}^{j}\right]\left.\right|^{2} \tau<C, \quad\left\|v^{n}\right\|^{2}+\sum_{j=1}^{n} \| v_{\bar{x}}^{j}\right]\left.\right|^{2} \tau<C, \quad n=1,2, \ldots, N
$$

where here and below $C$ is a positive constant independent from $\tau$ and $h$.

The a priori estimates (3.4) guarantee the stability of the scheme (3.1) - (3.3). Note, that applying the technique as we prove convergence theorem blow, it is not difficult to prove the uniqueness of the solution of the scheme (3.1) - (3.3) too.

The main statement of the present section can be stated as follows.

Theorem 2. If $a=a(S) \geq a_{0}=$ Const $>0, a^{\prime}(S) \geq 0, a^{\prime \prime}(S) \leq 0, g$ is monotonically increased function and problem (2.1) - (2.3) has a sufficiently smooth solution $(U(x, t), V(x, t))$, then the solution $u^{j}=\left(u_{1}^{j}, u_{2}^{j}, \ldots, u_{M-1}^{j}\right), v^{j}=\left(v_{1}^{j}, v_{2}^{j}, \ldots, v_{M-1}^{j}\right)$, $j=1,2, \ldots, N$ of the difference scheme (3.1) - (3.3) tends to the solution of continuous problem (2.1) - (2.3) $U^{j}=\left(U_{1}^{j}, U_{2}^{j}, \ldots, U_{M-1}^{j}\right), V^{j}=\left(V_{1}^{j}, V_{2}^{j}, \ldots, V_{M-1}^{j}\right)$, $j=1,2, \ldots, N$ as $\tau \rightarrow 0, h \rightarrow 0$ and the following estimates are true:

$$
\left\|u^{j}-U^{j}\right\| \leq C(\tau+h), \quad\left\|v^{j}-V^{j}\right\| \leq C(\tau+h) .
$$


Proof. Introducing the differences $z_{i}^{j}=u_{i}^{j}-U_{i}^{j}$ and $w_{i}^{j}=v_{i}^{j}-V_{i}^{j}$ we get the following relations:

$$
\begin{gathered}
z_{t, i}^{j+1}-\left\{a\left(\tau h \sum_{k=1}^{j+1} \sum_{\ell=1}^{M}\left[\left(u_{\bar{x}, \ell}^{k}\right)^{2}+\left(v_{\bar{x}, \ell}^{k}\right)^{2}\right]\right) u_{\bar{x}, i}^{j+1}\right. \\
\left.-a\left(\tau h \sum_{k=1}^{j+1} \sum_{\ell=1}^{M}\left[\left(U_{\bar{x}, \ell}^{k}\right)^{2}+\left(V_{\bar{x}, \ell}^{k}\right)^{2}\right]\right) U_{\bar{x}, i}^{j+1}\right\}_{x} \\
+g\left(u_{i}^{j+1}\right)-g\left(U_{i}^{j+1}\right)=-\psi_{1, i}^{j}, \\
w_{t, i}^{j+1}-\left\{a\left(\tau h \sum_{k=1}^{j+1} \sum_{\ell=1}^{M}\left[\left(u_{\bar{x}, \ell}^{k}\right)^{2}+\left(v_{\bar{x}, \ell}^{k}\right)^{2}\right]\right) v_{\bar{x}, i}^{j+1}\right. \\
\left.-a\left(\tau h \sum_{k=1}^{j+1} \sum_{\ell=1}^{M}\left[\left(U_{\bar{x}, \ell}^{k}\right)^{2}+\left(V_{\bar{x}, \ell}^{k}\right)^{2}\right]\right) V_{\bar{x}, i}^{j+1}\right\}_{x} \\
+g\left(v_{i}^{j+1}\right)-g\left(V_{i}^{j+1}\right)=-\psi_{2, i}^{j}, \\
z_{0}^{j}=w_{0}^{j}=z_{\bar{x}, M}^{j}=w_{\bar{x}, M}^{j}=0, \\
z_{i}^{0}=w_{i}^{0}=0,
\end{gathered}
$$

where $\psi_{1, i}^{j}$ and $\psi_{2, i}^{j}$ are approximation errors of scheme (3.1) and

$$
\psi_{k, i}^{j}=O(\tau+h), \quad k=1,2 .
$$

Multiplying the first equation of system (3.6) scalarly by the grid function $\tau z^{j+1}=$ $\tau\left(z_{1}^{j+1}, z_{2}^{j+1}, \ldots, z_{M-1}^{j+1}\right)$ and using the boundary conditions (3.7) we get

$$
\begin{gathered}
\left\|z^{j+1}\right\|^{2}-\left(z^{j+1}, z^{j}\right)+\tau h \sum_{i=1}^{M}\left\{a\left(\tau h \sum_{k=1}^{j+1} \sum_{\ell=1}^{M}\left[\left(u_{\bar{x}, \ell}^{k}\right)^{2}+\left(v_{\bar{x}, \ell}^{k}\right)^{2}\right]\right) u_{\bar{x}, i}^{j+1}\right. \\
\left.-a\left(\tau h \sum_{k=1}^{j+1} \sum_{\ell=1}^{M}\left[\left(U_{\bar{x}, \ell}^{k}\right)^{2}+\left(V_{\bar{x}, \ell}^{k}\right)^{2}\right]\right) U_{\bar{x}, i}^{j+1}\right\} z_{\bar{x}, i}^{j+1} \\
+\left(g\left(u_{i}^{j+1}\right)-g\left(U_{i}^{j+1}\right), u^{j+1}-U^{j+1}\right)=-\tau\left(\psi_{1}^{j}, z^{j+1}\right) .
\end{gathered}
$$


Analogously,

$$
\begin{gathered}
\left\|w^{j+1}\right\|^{2}-\left(w^{j+1}, w^{j}\right)+\tau h \sum_{i=1}^{M}\left\{a\left(\tau h \sum_{k=1}^{j+1} \sum_{\ell=1}^{M}\left[\left(u_{\bar{x}, \ell}^{k}\right)^{2}+\left(v_{\bar{x}, \ell}^{k}\right)^{2}\right]\right) v_{\bar{x}, i}^{j+1}\right. \\
\left.-a\left(\tau h \sum_{k=1}^{j+1} \sum_{\ell=1}^{M}\left[\left(U_{\bar{x}, \ell}^{k}\right)^{2}+\left(V_{\bar{x}, \ell}^{k}\right)^{2}\right]\right) V_{\bar{x}, i}^{j+1}\right\} w_{\bar{x}, i}^{j+1} \\
+\left(g\left(v_{i}^{j+1}\right)-g\left(V_{i}^{j+1}\right), v^{j+1}-V^{j+1}\right)=-\tau\left(\psi_{2}^{j}, w^{j+1}\right) .
\end{gathered}
$$

Adding these two equalities and taking into account monotonicity of the function $g$, from these two equalities we have

$$
\begin{gathered}
\left\|z^{j+1}\right\|^{2}-\left(z^{j+1}, z^{j}\right)+\left\|w^{j+1}\right\|^{2}-\left(w^{j+1}, w^{j}\right) \\
+\tau h \sum_{i=1}^{M}\left\{a\left(\tau h \sum_{k=1}^{j+1} \sum_{\ell=1}^{M}\left[\left(u_{\bar{x}, \ell}^{k}\right)^{2}+\left(v_{\bar{x}, \ell}^{k}\right)^{2}\right]\right) u_{\bar{x}, i}^{j+1}\right. \\
\left.-a\left(\tau h \sum_{k=1}^{j+1} \sum_{\ell=1}^{M}\left[\left(U_{\bar{x}, \ell}^{k}\right)^{2}+\left(V_{\bar{x}, \ell}^{k}\right)^{2}\right]\right) U_{\bar{x}, i}^{j+1}\right\} z_{\bar{x}, i}^{j+1} \\
+\tau h \sum_{i=1}^{M}\left\{a\left(\tau h \sum_{k=1}^{j+1} \sum_{\ell=1}^{M}\left[\left(u_{\bar{x}, \ell}^{k}\right)^{2}+\left(v_{\bar{x}, \ell}^{k}\right)^{2}\right]\right) v_{\bar{x}, i}^{j+1}\right. \\
\left.-a\left(\tau h \sum_{k=1}^{j+1} \sum_{\ell=1}^{M}\left[\left(U_{\bar{x}, \ell}^{k}\right)^{2}+\left(V_{\bar{x}, \ell}^{k}\right)^{2}\right]\right) V_{\bar{x}, i}^{j+1}\right\} w_{\bar{x}, i}^{j+1} \\
\leq-\tau\left(\psi_{1}^{j}, z^{j+1}\right)-\tau\left(\psi_{2}^{j}, w^{j+1}\right) .
\end{gathered}
$$

Note that, using the Hadamard formula

$$
\varphi(y)-\varphi(z)=\int_{0}^{1} \frac{d}{d \mu} \varphi[z+\mu(y-z)] d \mu,
$$

below we prove one of the main inequality to estimate terms with nonlinear diffusion coefficient $a(S)$

$$
\left\{a\left(\tau h \sum_{k=1}^{j+1} \sum_{\ell=1}^{M}\left[\left(u_{\bar{x}, \ell}^{k}\right)^{2}+\left(v_{\bar{x}, \ell}^{k}\right)^{2}\right]\right) u_{\bar{x}, i}^{j+1}\right.
$$




$$
\begin{aligned}
& \left.-a\left(\tau h \sum_{k=1}^{j+1} \sum_{\ell=1}^{M}\left[\left(U_{\bar{x}, \ell}^{k}\right)^{2}+\left(V_{\bar{x}, \ell}^{k}\right)^{2}\right]\right) U_{\bar{x}, i}^{j+1}\right\}\left(u_{\bar{x}, i}^{j+1}-U_{\bar{x}, i}^{j+1}\right) \\
& +\left\{a\left(\tau h \sum_{k=1}^{j+1} \sum_{\ell=1}^{M}\left[\left(u_{\bar{x}, \ell}^{k}\right)^{2}+\left(v_{\bar{x}, \ell}^{k}\right)^{2}\right]\right) v_{\bar{x}, i}^{j+1}\right. \\
& \left.-a\left(\tau h \sum_{k=1}^{j+1} \sum_{\ell=1}^{M}\left[\left(U_{\bar{x}, \ell}^{k}\right)^{2}+\left(V_{\bar{x}, \ell}^{k}\right)^{2}\right]\right) V_{\bar{x}, i}^{j+1}\right\}\left(v_{\bar{x}, i}^{j+1}-V_{\bar{x}, i}^{j+1}\right) \\
& =\int_{0}^{1}\left\{\frac{d}{d \mu} a\left(\tau h \sum_{k=1}^{j+1} \sum_{\ell=1}^{M}\left\{\left[U_{\bar{x}, \ell}^{k}+\mu\left(u_{\bar{x}, \ell}^{k}-U_{\bar{x}, \ell}^{k}\right)\right]^{2}+\left[V_{\bar{x}, \ell}^{k}+\mu\left(v_{\bar{x}, \ell}^{k}-V_{\bar{x}, \ell}^{k}\right)\right]^{2}\right\}\right)\right. \\
& \left.\times\left[U_{\bar{x}, i}^{j+1}+\mu\left(u_{\bar{x}, i}^{j+1}-U_{\bar{x}, i}^{j+1}\right)\right]\right\} d \mu\left(u_{\bar{x}, i}^{j+1}-U_{\bar{x}, i}^{j+1}\right) \\
& +\int_{0}^{1}\left\{\frac { d } { d \mu } a \left(\tau h \sum_{k=1}^{j+1} \sum_{\ell=1}^{M}\left\{\left[U_{\bar{x}, \ell}^{k}+\mu\left(u_{\bar{x}, \ell}^{k}-U_{\bar{x}, \ell}^{k}\right)\right]^{2}+\left[V_{\bar{x}, \ell}^{k}+\mu\left(v_{\bar{x}, \ell}^{k}-V_{\bar{x}, \ell}^{k}\right]^{2}\right\}\right)\right.\right. \\
& \left.\times\left[V_{\bar{x}, i}^{j+1}+\mu\left(v_{\bar{x}, i}^{j+1}-V_{\bar{x}, i}^{j+1}\right)\right]\right\} d \mu\left(v_{\bar{x}, i}^{j+1}-V_{\bar{x}, i}^{j+1}\right) \\
& =2 \int_{0}^{1} a^{\prime}\left(\tau h \sum_{k=1}^{j+1} \sum_{\ell=1}^{M}\left\{\left[U_{\bar{x}, \ell}^{k}+\mu\left(u_{\bar{x}, \ell}^{k}-U_{\bar{x}, \ell}^{k}\right)\right]^{2}+\left[V_{\bar{x}, \ell}^{k}+\mu\left(v_{\bar{x}, \ell}^{k}-V_{\bar{x}, \ell}^{k}\right)\right]^{2}\right\}\right) \\
& \times \tau h \sum_{k=1}^{j+1} \sum_{\ell=1}^{M}\left\{\left[U_{\bar{x}, \ell}^{k}+\mu\left(u_{\bar{x}, \ell}^{k}-U_{\bar{x}, \ell}^{k}\right)\right]\left(u_{\bar{x}, \ell}^{k}-U_{\bar{x}, \ell}^{k}\right)\right. \\
& \left.+\left[V_{\bar{x}, \ell}^{k}+\mu\left(v_{\bar{x}, \ell}^{k}-V_{\bar{x}, \ell}^{k}\right)\right]\left(v_{\bar{x}, \ell}^{k}-V_{\bar{x}, \ell}^{k}\right)\right\} \\
& \times\left[U_{\bar{x}, i}^{j+1}+\mu\left(u_{\bar{x}, i}^{j+1}-U_{\bar{x}, i}^{j+1}\right)\right] d \mu\left(u_{\bar{x}, i}^{j+1}-U_{\bar{x}, i}^{j+1}\right) \\
& +\int_{0}^{1} a\left(\tau h \sum_{k=1}^{j+1} \sum_{\ell=1}^{M}\left\{\left[U_{\bar{x}, \ell}^{k}+\mu\left(u_{\bar{x}, \ell}^{k}-U_{\bar{x}, i}^{k}\right)\right]^{2}+\left[V_{\bar{x}, \ell}^{k}+\mu\left(v_{\bar{x}, \ell}^{k}-V_{\bar{x}, \ell}^{k}\right)\right]^{2}\right\}\right) \\
& \times\left(u_{\bar{x}, i}^{j+1}-U_{\bar{x}, i}^{j+1}\right) d \mu\left(u_{\bar{x}, i}^{j+1}-U_{\bar{x}, i}^{j+1}\right) \\
& +2 \int_{0}^{1} a^{\prime}\left(\tau h \sum_{k=1}^{j+1} \sum_{\ell=1}^{M}\left\{\left[U_{\bar{x}, \ell}^{k}+\mu\left(u_{\bar{x}, \ell}^{k}-U_{\bar{x}, \ell}^{k}\right)\right]^{2}+\left[V_{\bar{x}, \ell}^{k}+\mu\left(v_{\bar{x}, \ell}^{k}-V_{\bar{x}, \ell}^{k}\right)\right]^{2}\right\}\right) \\
& \times \tau h \sum_{k=1}^{j+1} \sum_{\ell=1}^{M}\left\{\left[U_{\bar{x}, \ell}^{k}+\mu\left(u_{\bar{x}, \ell}^{k}-U_{\bar{x}, \ell}^{k}\right)\right]\left(u_{\bar{x}, \ell}^{k}-U_{\bar{x}, \ell}^{k}\right)\right. \\
& \left.+\left[V_{\bar{x}, \ell}^{k}+\mu\left(v_{\bar{x}, \ell}^{k}-V_{\bar{x}, \ell}^{k}\right)\right]\left(v_{\bar{x}, \ell}^{k}-V_{\bar{x}, \ell}^{k}\right)\right\}
\end{aligned}
$$




$$
\begin{aligned}
& \times\left[V_{\bar{x}, i}^{j+1}+\mu\left(v_{\bar{x}, i}^{j+1}-V_{\bar{x}, i}^{j+1}\right)\right] d \mu\left(v_{\bar{x}, i}^{j+1}-V_{\bar{x}, i}^{j+1}\right) \\
& +\int_{0}^{1} a\left(\tau h \sum_{k=1}^{j+1} \sum_{\ell=1}^{M}\left\{\left[U_{\bar{x}, \ell}^{k}+\mu\left(u_{\bar{x}, \ell}^{k}-U_{\bar{x}, \ell}^{k}\right)\right]^{2}+\left[V_{\bar{x}, \ell}^{k}+\mu\left(v_{\bar{x}, \ell}^{k}-V_{\bar{x}, \ell}^{k}\right)\right]^{2}\right\}\right) \\
& \times\left(v_{\bar{x}, \ell}^{j+1}-V_{\bar{x}, i}^{j+1}\right) d \mu\left(v_{\bar{x}, i}^{j+1}-V_{\bar{x}, i}^{j+1}\right) \\
& =2 \int_{0}^{1} a^{\prime}\left(\tau h \sum_{k=1}^{j+1} \sum_{\ell=1}^{M}\left\{\left[U_{\bar{x}, \ell}^{k}+\mu\left(u_{\bar{x}, \ell}^{k}-U_{\bar{x}, \ell}^{k}\right)\right]^{2}+\left[V_{\bar{x}, \ell}^{k}+\mu\left(v_{\bar{x}, \ell}^{k}-V_{\bar{x}, \ell}^{k}\right)\right]^{2}\right\}\right) \\
& \times \tau h \sum_{k=1}^{j+1} \sum_{\ell=1}^{M}\left\{\left[U_{\bar{x}, \ell}^{k}+\mu\left(u_{\bar{x}, \ell}^{k}-U_{\bar{x}, \ell}^{k}\right)\right]\left(u_{\bar{x}, \ell}^{k}-U_{\bar{x}, \ell}^{k}\right)\right. \\
& \left.+\left[V_{\bar{x}, \ell}^{k}+\mu\left(v_{\bar{x}, \ell}^{k}-V_{\bar{x}, \ell}^{k}\right)\right]\left(v_{\bar{x}, \ell}^{k}-V_{\bar{x}, \ell}^{k}\right)\right\} \\
& \times\left\{\left[U_{\bar{x}, i}^{j+1}+\mu\left(u_{\bar{x}, i}^{j+1}-U_{\bar{x}, i}^{j+1}\right)\right]\left(u_{\bar{x}, i}^{j+1}-U_{\bar{x}, i}^{j+1}\right)\right. \\
& \left.+\left[V_{\bar{x}, i}^{j+1}+\mu\left(v_{\bar{x}, i}^{j+1}-V_{\bar{x}, i}^{j+1}\right)\right]\left(v_{\bar{x}, i}^{j+1}-V_{\bar{x}, i}^{j+1}\right)\right\} d \mu \\
& +\int_{0}^{1} a\left(\tau h \sum_{k=1}^{j+1} \sum_{\ell=1}^{M}\left\{\left[U_{\bar{x}, \ell}^{k}+\mu\left(u_{\bar{x}, \ell}^{k}-U_{\bar{x}, i}^{k}\right)\right]^{2}+\left[V_{\bar{x}, \ell}^{k}+\mu\left(v_{\bar{x}, \ell}^{k}-V_{\bar{x}, \ell}^{k}\right)\right]^{2}\right\}\right) \\
& \times\left[\left(u_{\bar{x}, i}^{j+1}-U_{\bar{x}, i}^{j+1}\right)^{2}+\left(v_{\bar{x}, i}^{j+1}-V_{\bar{x}, i}^{j+1}\right)^{2}\right] d \mu \\
& =2 \int_{0}^{1} a^{\prime}\left(\tau h \sum_{k=1}^{j+1} \sum_{\ell=1}^{M}\left\{\left[U_{\bar{x}, \ell}^{k}+\mu\left(u_{\bar{x}, \ell}^{k}-U_{\bar{x}, \ell}^{k}\right)\right]^{2}+\left[V_{\bar{x}, \ell}^{k}+\mu\left(v_{\bar{x}, \ell}^{k}-V_{\bar{x}, \ell}^{k}\right)\right]^{2}\right\}\right) \xi^{j+1}(\mu) \xi_{t}^{j}(\mu) d \mu \\
& +\int_{0}^{1} a\left(\tau h \sum_{k=1}^{j+1} \sum_{\ell=1}^{M}\left\{\left[U_{\bar{x}, \ell}^{k}+\mu\left(u_{\bar{x}, \ell}^{k}-U_{\bar{x}, \ell}^{k}\right)\right]^{2}+\left[V_{\bar{x}, \ell}^{k}+\mu\left(v_{\bar{x}, \ell}^{k}-V_{\bar{x}, \ell}^{k}\right)\right]^{2}\right\}\right) \\
& \times\left[\left(u_{\bar{x}, i}^{j+1}-U_{\bar{x}, i}^{j+1}\right)^{2}+\left(v_{\bar{x}, i}^{j+1}-V_{\bar{x}, i}^{j+1}\right)^{2}\right] d \mu,
\end{aligned}
$$

where

$$
\begin{gathered}
\xi^{j+1}(\mu)=\tau h \sum_{k=1}^{j+1} \sum_{\ell=1}^{M}\left\{\left[U_{\bar{x}, \ell}^{k}+\mu\left(u_{\bar{x}, \ell}^{k}-U_{\bar{x}, \ell}^{k}\right)\right]\left(u_{\bar{x}, \ell}^{k}-U_{\bar{x}, \ell}^{k}\right)\right. \\
\left.+\left[V_{\bar{x}, \ell}^{k}+\mu\left(v_{\bar{x}, \ell}^{k}-V_{\bar{x}, \ell}^{k}\right)\right]\left(v_{\bar{x}, \ell}^{k}-V_{\bar{x}, \ell}^{k}\right)\right\}, \\
\xi^{0}(\mu)=0,
\end{gathered}
$$


and therefore,

$$
\begin{aligned}
\xi_{t}^{j}(\mu) & =h \sum_{\ell=1}^{M}\left[U_{\bar{x}, \ell}^{j+1}+\mu\left(u_{\bar{x}, \ell}^{j+1}-U_{\bar{x}, \ell}^{j+1}\right)\right]\left(u_{\bar{x}, \ell}^{j+1}-U_{\bar{x}, \ell}^{j+1}\right) \\
& +\left[V_{\bar{x}, \ell}^{j+1}+\mu\left(v_{\bar{x}, \ell}^{j+1}-V_{\bar{x}, \ell}^{j+1}\right)\right]\left(v_{\bar{x}, \ell}^{j+1}-V_{\bar{x}, \ell}^{j+1}\right) .
\end{aligned}
$$

Introducing the following notation

$$
s^{j+1}(\mu)=\tau h \sum_{k=1}^{j+1} \sum_{\ell=1}^{M}\left\{\left[U_{\bar{x}, \ell}^{k}+\mu\left(u_{\bar{x}, \ell}^{k}-U_{\bar{x}, \ell}^{k}\right)\right]^{2}+\left[V_{\bar{x}, \ell}^{k}+\mu\left(v_{\bar{x}, \ell}^{k}-V_{\bar{x}, \ell}^{k}\right)\right]^{2}\right\},
$$

from the previous equality we have

$$
\begin{gathered}
\left\{a\left(\tau h \sum_{k=1}^{j+1} \sum_{\ell=1}^{M}\left[\left(u_{\bar{x}, \ell}^{k}\right)^{2}+\left(v_{\bar{x}, \ell}^{k}\right)^{2}\right]\right) u_{\bar{x}, i}^{j+1}\right. \\
\left.-a\left(\tau h \sum_{k=1}^{j+1} \sum_{\ell=1}^{M}\left[\left(U_{\bar{x}, \ell}^{k}\right)^{2}+\left(V_{\bar{x}, \ell}^{k}\right)^{2}\right]\right) U_{\bar{x}, i}^{j+1}\right\}\left(u_{\bar{x}, i}^{j+1}-U_{\bar{x}, i}^{j+1}\right) \\
+\left\{a\left(\tau h \sum_{k=1}^{j+1} \sum_{\ell=1}^{M}\left[\left(u_{\bar{x}, \ell}^{k}\right)^{2}+\left(v_{\bar{x}, \ell}^{k}\right)^{2}\right]\right) v_{\bar{x}, i}^{j+1}\right. \\
-a\left(\tau h \sum_{k=1}^{j+1} \sum_{\ell=1}^{M}\left[\left(U_{\bar{x}, \ell}^{k}\right)^{2}+\left(V_{\bar{x}, \ell}^{k}\right)^{2}\right] V_{\bar{x}, i}^{j+1}\right\}\left(v_{\bar{x}, i}^{j+1}-V_{\bar{x}, i}^{j+1}\right) \\
=2 \int_{0}^{1} a^{\prime}\left(s^{j+1}(\mu)\right) \xi^{j+1} \xi_{t}^{j} d \mu \\
+\int_{0}^{1} a\left(s^{j+1}(\mu)\right)\left[\left(u_{\bar{x}, i}^{j+1}-U_{\bar{x}, i}^{j+1}\right)^{2}+\left(v_{\bar{x}, i}^{j+1}-V_{\bar{x}, i}^{j+1}\right)^{2}\right] d \mu .
\end{gathered}
$$


After substituting this equality in (3.9) we get

$$
\begin{gathered}
\left\|z^{j+1}\right\|^{2}-\left(z^{j+1}, z^{j}\right)+\left\|w^{j+1}\right\|^{2}-\left(w^{j+1}, w^{j}\right) \\
+2 \tau h \sum_{i=1}^{M} \int_{0}^{1} a^{\prime}\left(s^{j+1}(\mu)\right) \xi^{j+1} \xi_{t}^{j} d \mu \\
+\tau h \sum_{i=1}^{M} \int_{0}^{1} a\left(s^{j+1}(\mu)\right)\left[\left(u_{\bar{x}, i}^{j+1}-U_{\bar{x}, i}^{j+1}\right)^{2}+\left(v_{\bar{x}, i}^{j+1}-V_{\bar{x}, i}^{j+1}\right)^{2}\right] d \mu \\
\leq-\tau\left(\psi_{1}^{j}, z^{j+1}\right)-\tau\left(\psi_{2}^{j}, w^{j+1}\right) .
\end{gathered}
$$

Taking into account that $a(S) \geq a_{0}=$ Const $>0$ and relations $s^{j+1}(\mu) \geq 0$,

$$
\begin{gathered}
\left(r^{j+1}, r^{j}\right)=\frac{1}{2}\left\|r^{j+1}\right\|^{2}+\frac{1}{2}\left\|r^{j}\right\|^{2}-\frac{1}{2}\left\|r^{j+1}-r^{j}\right\|^{2}, \\
\tau \xi^{j+1} \xi_{t}^{j}=\frac{1}{2}\left(\xi^{j+1}\right)^{2}-\frac{1}{2}\left(\xi^{j}\right)^{2}+\frac{\tau^{2}}{2}\left(\xi_{t}^{j}\right)^{2}
\end{gathered}
$$

from (3.10) we have

$$
\begin{gathered}
\left\|z^{j+1}\right\|^{2}-\frac{1}{2}\left\|z^{j+1}\right\|^{2}-\frac{1}{2}\left\|z^{j}\right\|^{2}+\frac{1}{2}\left\|z^{j+1}-z^{j}\right\|^{2} \\
+\left\|w^{j+1}\right\|^{2}-\frac{1}{2}\left\|w^{j+1}\right\|^{2}-\frac{1}{2}\left\|w^{j}\right\|^{2}+\frac{1}{2}\left\|w^{j+1}-w^{j}\right\|^{2} \\
+h \sum_{i=1}^{M} \int_{0}^{1} a^{\prime}\left(s^{j+1}(\mu)\right)\left[\left(\xi^{j+1}\right)^{2}-\left(\xi^{j}\right)^{2}\right] d \mu \\
+\tau^{2} h \sum_{i=1}^{M} \int_{0}^{1} a^{\prime}\left(s^{j+1}(\mu)\right)\left(\xi_{t}^{j}\right)^{2} d \mu \\
+\tau h a_{0} \sum_{i=1}^{M}\left[\left(u_{\bar{x}, i}^{j+1}-U_{\bar{x}, i}^{j+1}\right)^{2}+\left(v_{\bar{x}, i}^{j+1}-V_{\bar{x}, i}^{j+1}\right)^{2}\right] \\
\leq-\tau\left(\psi_{1}^{j}, z^{j+1}\right)-\tau\left(\psi_{2}^{j}, w^{j+1}\right) .
\end{gathered}
$$


From (3.11) we arrive at

$$
\begin{gathered}
\frac{1}{2}\left\|z^{j+1}\right\|^{2}-\frac{1}{2}\left\|z^{j}\right\|^{2}+\frac{\tau^{2}}{2}\left\|z_{t}^{j}\right\|^{2} \\
+\frac{1}{2}\left\|w^{j+1}\right\|^{2}-\frac{1}{2}\left\|w^{j}\right\|^{2}+\frac{\tau^{2}}{2}\left\|w_{t}^{j}\right\|^{2} \\
+h \sum_{i=1}^{M} \int_{0}^{1} a^{\prime}\left(s^{j+1}(\mu)\right)\left[\left(\xi^{j+1}\right)^{2}-\left(\xi^{j}\right)^{2}\right] d \mu \\
\left.\left.+\left.\tau a_{0}\left(\| z_{\bar{x}}^{j+1}\right]\right|^{2}+\| w_{\bar{x}}^{j+1}\right]\left.\right|^{2}\right) \leq \frac{\tau}{2 a_{0}}\left(\left\|\psi_{1}^{j}\right\|^{2}+\left\|\psi_{2}^{j}\right\|^{2}\right) \\
+\frac{\tau a_{0}}{2}\left(\left\|z^{j+1}\right\|^{2}+\left\|w^{j+1}\right\|^{2}\right) .
\end{gathered}
$$

Using discrete analogue of Poincar inequality [21]

$$
\left.\left\|r^{j+1}\right\|^{2} \leq \| r_{\bar{x}}^{j+1}\right]\left.\right|^{2},
$$

from (3.12) we get

$$
\begin{gathered}
\left\|z^{j+1}\right\|^{2}-\left\|z^{j}\right\|^{2}+\tau^{2}\left\|z_{t}^{j}\right\|^{2}+\left\|w^{j+1}\right\|^{2}-\left\|w^{j}\right\|^{2}+\tau^{2}\left\|w_{t}^{j}\right\|^{2} \\
+2 h \sum_{i=1}^{M} \int_{0}^{1} a^{\prime}\left(s^{j+1}(\mu)\right)\left[\left(\xi^{j+1}\right)^{2}-\left(\xi^{j}\right)^{2}\right] d \mu \\
\left.+\left.\tau a_{0}\left(\| z_{\bar{x}}^{j+1}\right]\right|^{2}+\left\|w_{\bar{x}}^{j+1}\right\|^{2}\right) \leq \frac{\tau}{a_{0}}\left(\left\|\psi_{1}^{j}\right\|^{2}+\left\|\psi_{2}^{j}\right\|^{2}\right) .
\end{gathered}
$$

Summing (3.13) from $j=0$ to $j=n-1$ we arrive at

$$
\begin{gathered}
\left\|z^{n}\right\|^{2}+\tau^{2} \sum_{j=0}^{n-1}\left\|z_{t}^{j}\right\|^{2}+\left\|w^{n}\right\|^{2}+\tau^{2} \sum_{j=0}^{n-1}\left\|w_{t}^{j}\right\|^{2} \\
+2 h \sum_{j=0}^{n-1} \sum_{i=1}^{M} \int_{0}^{1} a^{\prime}\left(s^{j+1}(\mu)\right)\left[\left(\xi^{j+1}\right)^{2}-\left(\xi^{j}\right)^{2}\right] d \mu \\
\left.\left.+\left.\tau a_{0} \sum_{j=0}^{n-1}\left(\| z_{\bar{x}}^{j+1}\right]\right|^{2}+\| w_{\bar{x}}^{j+1}\right]\left.\right|^{2}\right) \leq \frac{\tau}{a_{0}} \sum_{j=0}^{n-1}\left(\left\|\psi_{1}^{j}\right\|^{2}+\left\|\psi_{2}^{j}\right\|^{2}\right) .
\end{gathered}
$$


Note, that since $s^{j+1}(\mu) \geq s^{j}(\mu), a^{\prime}(S) \geq 0$ and $a^{\prime \prime}(S) \leq 0$, for the second line of last formula we have

$$
\begin{gathered}
\sum_{j=0}^{n-1} a^{\prime}\left(s^{j+1}(\mu)\right)\left[\left(\xi^{j+1}\right)^{2}-\left(\xi^{j}\right)^{2}\right] \\
=a^{\prime}\left(s^{1}(\mu)\right)\left(\xi^{1}\right)^{2}-a^{\prime}\left(s^{1}(\mu)\right)\left(\xi^{0}\right)^{2} \\
+a^{\prime}\left(s^{2}(\mu)\right)\left(\xi^{2}\right)^{2}-a^{\prime}\left(s^{2}(\mu)\right)\left(\xi^{1}\right)^{2} \\
+\cdots+a^{\prime}\left(s^{n}(\mu)\right)\left(\xi^{n}\right)^{2}-a^{\prime}\left(s^{n}(\mu)\right)\left(\xi^{n-1}\right)^{2} \\
=a^{\prime}\left(s^{n}(\mu)\right)\left(\xi^{n}\right)^{2}+\sum_{j=1}^{n-1}\left[a^{\prime}\left(s^{j}(\mu)\right)-a^{\prime}\left(s^{j+1}(\mu)\right)\right]\left(\xi^{j}\right)^{2} \geq 0 .
\end{gathered}
$$

Taking into account the last relation and (3.14) one can deduce

$$
\begin{gathered}
\left\|z^{n}\right\|^{2}+\left\|w^{n}\right\|^{2}+\tau^{2} \sum_{j=0}^{n-1}\left\|z_{t}^{j}\right\|^{2}+\tau^{2} \sum_{j=0}^{n-1}\left\|w_{t}^{j}\right\|^{2} \\
\left.\left.+\left.\tau a_{0} \sum_{j=0}^{n-1}\left(\| z_{\bar{x}}^{j+1}\right]\right|^{2}+\| w_{\bar{x}}^{j+1}\right]\left.\right|^{2}\right) \leq \frac{\tau}{a_{0}} \sum_{j=0}^{n-1}\left(\left\|\psi_{1}^{j}\right\|^{2}+\left\|\psi_{2}^{j}\right\|^{2}\right) .
\end{gathered}
$$

From (3.15) we get (3.5), and thus Theorem 2 is proved.

\section{Conclusion}

Uniqueness and large time behavior of solution of initial-boundary value problem with mixed boundary conditions as well as finite difference scheme for one nonlinear partial integro-differential averaged model with source terms are investigated. Mentioned model is based on Maxwell system which describes electromagnetic field penetration into a medium whose electro-conductivity depends on temperature. Theorem of uniqueness and stabilization of solution is fixed. Convergence of the fully discrete scheme is proved. Wider class of nonlinearity considering more general cases of the diffusion coefficient $a=a(S)$ is studied.

\section{ACKNOWLEDGEMENT}

The third author thanks Shota Rustaveli National Science Foundation (project PhDF2016_19) for the financial support. 


\section{REFERENCES}

[1] Y. Bai and P. Zhang, "On a class of volterra nonlinear equations of parabolic type." Applied Mathematics and Computation, vol. 216, no. 1, pp. 236-240, 2010, doi: 10.1016/j.amc.2010.01.044.

[2] F. Chen, "Crank-Nicolson fully discrete Galerkin mixed finite element approximation of one nonlinear integrodifferential model." Abstract and Applied Analysis, vol. 2014, pp. 8, Article ID 534 902, 2014, doi: 10.1155/2014/534902.

[3] T. A. Dzhangveladze, "First boundary value problem for a nonlinear equation of parabolic type." Soviet Physics Doklady, vol. 28, pp. 323-324, 1983, doi: http://adsabs.harvard.edu/abs/1983SPhD...28..323D.

[4] T. A. Dzhangveladze, "A nonlinear integro-differential equations of parabolic type." Differential Equations, vol. 21, no. 1, pp. 32-36, 1985, doi: http://www.ams.org/mathscinetgetitem? $\mathrm{mr}=777778$.

[5] D. G. Gordeziani, T. A. Dzhangveladze, and T. K. Korshia, "Existence and uniqueness of a solution of certain nonlinear parabolic problems." Differential Equations, vol. 19, pp. 887-895, 1983, doi: http://www.ams.org/mathscinet-getitem?mr=708616.

[6] G. Gripenberg, S.-O. Londen, and O. Staffans, Volterra Integral and Functional Equations. Cambridge University Press, Cambridge, 1990. doi: 10.1017/CBO9780511662805.

[7] T. Jangveladze, Z. Kiguradze, and B. Neta, "Large time asymptotic and numerical solution of a nonlinear diffusion model with memory." Computers \& Mathematics with Applications, vol. 59, no. 1, pp. 254-273, 2010, doi: 10.1016/j.camwa.2009.07.052.

[8] T. Jangveladze, Z. Kiguradze, and B. Neta, Numerical Solution of Three Classes of Nonlinear Parabolic Integro-Differential Equations. Elsevier, Academic Press, 2016. doi: http://www.sciencedirect.com/science/book/9780128046289.

[9] T. A. Jangveladze, "Convergence of a difference scheme for a nonlinear integro-differential equation." Proceedings of I. Vekua Institute of Applied Mathematics, vol. 48, pp. 38-43, 1998, doi: 10.1016/j.amc.2014.02.021.

[10] T. A. Jangveladze and Z. V. Kiguradze, "Asymptotics for large time of solutions to nonlinear system associated with the penetration of a magnetic field into a substance." Application of Mathematics, vol. 55, pp. 471-493, 2010, doi: 10.1007/s10492-010-0019-3.

[11] Z. Kiguradze, "On one two-dimensional nonlinear integro-differential equation." International Workshop QUALITDE - 2015., pp. 83-85, 2015, doi: http://rmi.tsu.ge/eng/QUALITDE2015/Kiguradze_z_workshop_2015.pdf.

[12] L. Landau and E. Lifschitz, Electrodynamics of continuous media, Course of Theoretical Physics. Oxford, London, New York, Paris: Pergamon Press, 1957. doi: https://archive.org/details/ElectrodynamicsOfContinuousMedia.

[13] G. Laptev, "Quasilinear parabolic equations which contains in coefficients volterra's operator." Matematicheskii Sbornik, vol. 64, no. 2, pp. 527-542, 1989, doi: http://stacks.iop.org/00255734/64/i=2/a=A16.

[14] G. Laptev, "Mathematical features of the problem of the penetration of a magnetic field into matter." USSR Computational Mathematics and Mathematical Physics, vol. 28, pp. 35-45, 1990, doi: 10.1016/0041-5553(88)90005-5.

[15] H.-L. Liao and Y. Zhao, "Linearly localized difference schemes for the nonlinear Maxwell model of a magnetic field into a substance." Applied Mathematics and Computation, vol. 233, pp. 608622, 2014, doi: 10.1016/j.amc.2014.02.021.

[16] Y. Lin and H. M. Yin, "Nonlinear parabolic equations with nonlinear functionals." Journal of Mathematical Analysis and Applications, vol. 168, pp. 28-41, 1992, doi: 10.1016/0022247X(92)90187-I. 
[17] J.-L. Lions, Quelques Methodes de Resolution des Problemes aux Limites Non-lineaires. Dunod Gauthier-Villars, Paris, 1969. doi: http://www.worldcat.org/title/quelques-methodes-deresolution-des-problemes-aux-limites-non-lineaires/oclc/268273.

[18] N. Long and A. Dinh, "Nonlinear parabolic problem associated with the penetration of a magnetic field into a substance." Mathematical Methods in the Applied Sciences, vol. 16, pp. 281-295, 1993, doi: 10.1002/mma.1670160404.

[19] O. Pironneau, "Computer solutions of Maxwell's equations in homogeneous media." International Journal for Numerical Methods in Fluids, vol. 43, pp. 823-838, 2003, doi: 10.1002/fld.502.

[20] E. Purcell, Electricity and Magnetism. 2nd ed. M.: Nauka, 1975. doi: 10.1080/00107514.2012.661792.

[21] A. A. Samarskii, The Theory of Difference Schemes, (Russian). Nauka, Moscow, $1977 . \quad$ doi: http://samarskii.ru/books/book2001_2.pdf.

[22] N. Sharma, M. Khebchareon, K. Sharma, and A. Pani, "Finite element Galerkin approximations to a class of nonlinear and nonlocal parabolic problems." Numerical Methods for Partial Differential Equations, vol. 32, no. 4, pp. 1232-1264, 2016, doi: 10.1002/num.22048.

[23] N. Sharma and K. K. Sharma, "Unconditionally stable numerical method for a nonlinear partial integro-differential equation." Computers and Mathematics with Applications, vol. 67, pp. 62-76, 2014, doi: 10.1016/j.camwa.2013.10.010.

[24] N. Sharma and K. K. Sharma, "Finite element method for a nonlinear parabolic integro-differential equation in higher spatial dimensions." Applied Mathematical Modelling, vol. 39, pp. 7338-7350, 2015, doi: 10.1016/j.apm.2015.02.037.

Authors' addresses

T. Jangveladze

Georgian Technical University, Department of Mathematics, 77 Kostava Avenue, 0175 Tbilisi, Georgia

Current address: Ivane Javakhishvili Tbilisi State University, Ilia Vekua Institute of Applied Mathematics, 2 University Street, 0186 Tbilisi, Georgia

E-mail address: t.jangveladze@gtu.ge; temur.jangveladze@tsu.ge

\section{Z. Kiguradze}

Georgian Technical University, Department of Mathematics, 77 Kostava Avenue, 0175 Tbilisi, Georgia

Current address: Missouri University of Science and Technology, Department of Electrical and Computer Engineering, EMC Laboratory, 4000 Enterprise Drive, 65409 Rolla, MO, USA

E-mail address: kiguradzzemst.edu; z.kiguradze@gtu.ge

M. Kratsashvili

Sokhumi State University, Department of Mathematics and Computer Sciences, 12 Politkovskaia Street, 0186 Tbilisi, Georgia

E-mail address: maiakratsashvili@gmail.com 